Research Paper

\title{
Prognostic Factors among Brain Metastases in Newly Diagnosed Ovary Cancer: A Large Real-world Study
}

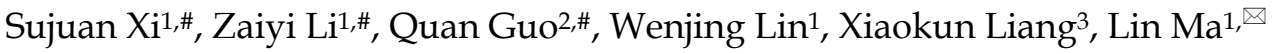 \\ 1. The Reproductive Medical Center, The Seventh Affiliated Hospital of Sun Yat-sen University, Shenzhen 518107, China \\ 2. Department of gynaechology, Shengjing Hospital Medical University, Shenyang, China \\ 3. Shenzhen Colleges of Advanced Technology, University of Chinese Academy of Sciences, Shenzhen, Guangdong, 518055 China \\ \# The authors contributed equally to this work. \\ $\square$ Corresponding author: Lin Ma, The Reproductive Medical Center, The Seventh Affiliated Hospital of Sun Yat-sen University, No.628, Zhenyuan Road, Xinhu \\ Street, Guangming District, Shenzhen, China; Tel: +86 1390 3056356. E-mail addresses: malin8@mail.sysu.edu.cn (L. Ma). \\ (C) The author(s). This is an open access article distributed under the terms of the Creative Commons Attribution License (https://creativecommons.org/licenses/by/4.0/). \\ See http://ivyspring.com/terms for full terms and conditions.
}

Received: 2020.02.02; Accepted: 2020.04.27; Published: 2020.05.18

\begin{abstract}
Background: Population-based data on the prognosis of brain metastases at initial diagnosis of ovary cancer (OCBM) are currently lacking. Besides, the effective treatment for OCBM patients is still controversial now. The study aimed to explore the prognostic factors among OCBM.

Methods: We retrospectively reviewed the OCBM patients from the Surveillance, Epidemiology, and End Result (SEER) database of the National Cancer Institute to investigate predictors of the presence of OCBM and its' prognostic factors related to all-cause mortality. We employed multivariable logistic and Cox regression analysis. Furthermore, to minimize the impact of potential confounding factors, we conducted a 1:1 propensity score matching (PSM) analysis.

Results: A total of 29,512 cases of OC patients entered into the study, including 89 patients with brain metastases of ovarian cancer, which accounted for $0.30 \%$ of the entire cohort and $12.02 \%$ of the metastatic disease subset. We identified eight factors, including laterality, histology, surgery, radiotherapy, chemotherapy, and extracranial metastatic sites to bone, liver, and lung, as predictors of OCBM based on multivariable logistic regression among the entire cohort. The median survival time of OCBM was 2.0 months, and the interquartile range was 2.0-10.0 mo. The patients who received comprehensive treatment had better prognosis. Based on the multivariable Cox model, marital status, surgery, chemotherapy, and extensive therapy (including RSC, SC, and RC) were identified as predictors of OS. Besides, a new factor (brain metastasis) was identified by 1:1 PSM -based multiple Cox regression, apart from the above prognostic factors for OS.

Conclusions: This study provided a population-based estimate of the proportion and prognosis for newly diagnosed ovary cancer with brain metastases. These findings may add materials to guidelines for preliminary screening and optimal treatment of OCBM patients.
\end{abstract}

Key words: Ovarian cancer; Brain metastases; Prognosis; associated factor; SEER

\section{Introduction}

Ovarian cancer (OC) accounts for about $2.5 \%$ of total malignancies among women, however, causing nearly $5 \%$ of all cancer-related deaths due to its high mortality rate (1-3). In the year of 2018, there are approximately 22,240 newly diagnosed ovary cancer cases, and about 14,070 estimated deaths occurred (2). OC patients were often found at an advanced stage (III or IV) when diagnosed, taking up over $60 \%$ of the total number, with synchronous distant metastases (4). That might partially explain their higher mortality (5). According to the latest study, the brain ranked the fifth common metastatic site behind the liver, distant lymph nodes, lung, and bone (6). Brain metastases from ovary cancer always portend a grave prognosis, leaving only a few months of survival after diagnosis (7). The incidence of ovarian carcinoma with brain 
metastases (OCBM) was reported to range from $0.29 \%$ to $12.0 \%(8-13)$.

However, clinical data on ovarian cancer brain lesions are scarce. The scarcity and low prevalence of OCBM make it challenging to draw firm conclusions or reach a consensus on the optimal therapy. Moreover, the brain remains a "sanctuary site" for the complex blood-brain barrier structure, limiting the penetration of drugs and causing problems for treatment. Treatment strategies are still controversial, and their efficiency needs further evaluation. Besides, there are still many things unknown about the predictors as well as prognostic factors of OCBM. There is a lack of reliable and multicentral population-based series study of the incidence of brain metastases in ovary cancer diagnosis (14). Based on the SEER database, this article focused on the risk and prognostic factors of de novo brain metastases in ovarian carcinoma.

\section{Materials and Methods}

\section{Database}

The SEER database has recorded cancer data of 18 population-based cancer registries, covering nearly $30 \%$ of the U.S. population $(15,16)$. It has collected information on patient clinical demographics, primary tumor site, tumor morphology and stage at diagnosis, the first course of therapy, and follow-up for vital status (https://seer.cancer.gov/about/ overview.html). We employed the SEERStat software to determine eligible patients for analysis, and extracted the information on cancer incidence from the official website (https://seer.cancer.gov/). Since the SEER database began to record metastatic information including brain metastases from the year of 2010. We collected the information about OCBM from 1 January 2010 to 31 December 2016.

\section{Study population}

In the SEER database, a total of 40,860 patients were diagnosed with ovarian cancer, excluding 395 less than 18 years old. Among them, we identified 38,021 patients with clear brain metastasis information. We excluded those patients with other cancers, or unknown sequence numbers, or diagnosed by autopsy or via death from the analysis, leaving 29,512 active follow-up patients in the final cohort for further analysis. Of these, 3546 patients diagnosed with metastatic disease to any distant site, and 89 patients diagnosed with OCBM.

In the entire cohort, the percentage of metastatic disease was $12.02 \%$, and the brain metastasis was $0.30 \%$. The data extraction flowchart was shown in Figure 1. The inclusion criteria were as follows: age elder than 18 years old; ovary cancer as the only primary malignant tumor; with clear information on brain metastases; with active follow-up. Exclusion criteria included the following requirements: age younger than 18 years old at diagnosis; with cancer other than ovary cancer; no clear information about brain metastases; diagnosis based on the death certificate or autopsy; no definite survival time; no active follow-up.

\section{Statistical analysis}

The baseline characteristics of the population were shown in Table 1 . We stratified the patients by age (18-58, 59+ years old), race (white, black, others and unknown), origin recode (Spanish-HispanicLatino and Non-Spanish-Hispanic-Latino), laterality (unilateral including left, right, only one side unspecified, bilateral and unknown), sequence of radiotherapy and surgery (radiotherapy before surgery, radiotherapy after surgery, radiotherapy before and after surgery and others), treatment and other sociodemographic information, such as marital status (married, single, divorced, widowed and unknown), insurance situation (yes, no and unknown), residence type (rural, urban, metropolitan and unknown). Based upon the 7th edition of the American Joint Committee on Cancer (AJCC) Cancer Staging Manual, we classified T staging (T1, T2, T3 and unknown) and $\mathrm{N}$ staging (N0, N1 and unknown). Tumor staging was classified into 5 categories: I, II, III, IV and unknown, according to the $7^{\text {th }}$ edition of the AJCC Cancer Staging Manual and SEER combined stage group as well. Surgery was classified as unilateral/bilateral (salpingo-) oophorectomy (25-28, 35-37, 50-52, 55-57, 80); debulking/cytoreductive surgery $(60-63)$, others $(17,70-74,90)$ and no surgery $(0,99)$. We reclassified treatment into 8 categories: patients receiving all three therapeutic approaches including chemotherapy, surgery and radiotherapy(CSR), radiotherapy and surgery (RS), chemotherapy and surgery (SC), radiotherapy and chemotherapy $(\mathrm{RC})$, only receiving radiotherapy $(\mathrm{R})$, only receiving surgery (S), only receiving chemotherapy $(\mathrm{C})$ and patients received none of any treatment above (Others) as other investigators(16). We defined the resident type by the county attributes from the 2003 US Department of Agriculture rural-urban continuum codes.

We stratified baseline clinical data (Table 1) and calculated the incidence proportion of the OCBM patients among the metastatic disease cohort and the entire cohort as well. Besides, we employed univariable and multivariable logistic regression analysis to identify potential predictors for the incidence of brain metastases at the diagnosis of OC. Survival estimates were based on the Kaplan-Meier 
method. Univariate and multivariate Cox regression analysis were employed to identify potential covariates associated with increased all-cause mortality. In the Cox regression model, we constructed 2 models for analysis. The first model included the following variables: marital status, histology, surgery, radiotherapy, chemotherapy, and insurance type. In the second model, we used treatment (CSR, RS, SC, RC, R, S, C, Others) to replace three therapeutic variables (surgery, radiotherapy, chemotherapy), and keep other variables the same with the model 1, as other investigators(16).

Additionally, to further control the potential confounding factors across groups, we employed a 1:1 propensity-score matching (PSM) analysis based on whether brain met or not to re-examine the impact of brain metastasis among ovarian cancer on the overall survival. In this study, a 1:1 pair matching was performed by the nearest neighbor method to generate a matched pair among the brain met group and without brain met group. A chi-square test was employed to compare categorical variables across groups.

The statistical analyses were performed using SPSS statistical software (version 22.0) and RStutio (version 1.1.453). A two-sided $p$-value $<0.05$ was considered statistically significant.

\section{Results}

\section{Patient characteristics and Proportion}

A total of 29,512 patients diagnosed with ovary cancer entered into our study, including 89 patients diagnosed with OCBM, and the median age was 62 . The clinical baseline characteristics were demonstrated in Table 1. Among 29,512 patients diagnosed with ovary cancer between 2010 and 2016 in the U.S., $3546(12.02 \%)$ presented with metastatic disease, and $89(0.30 \%)$ patients presented with synchronous brain metastases when diagnosed.

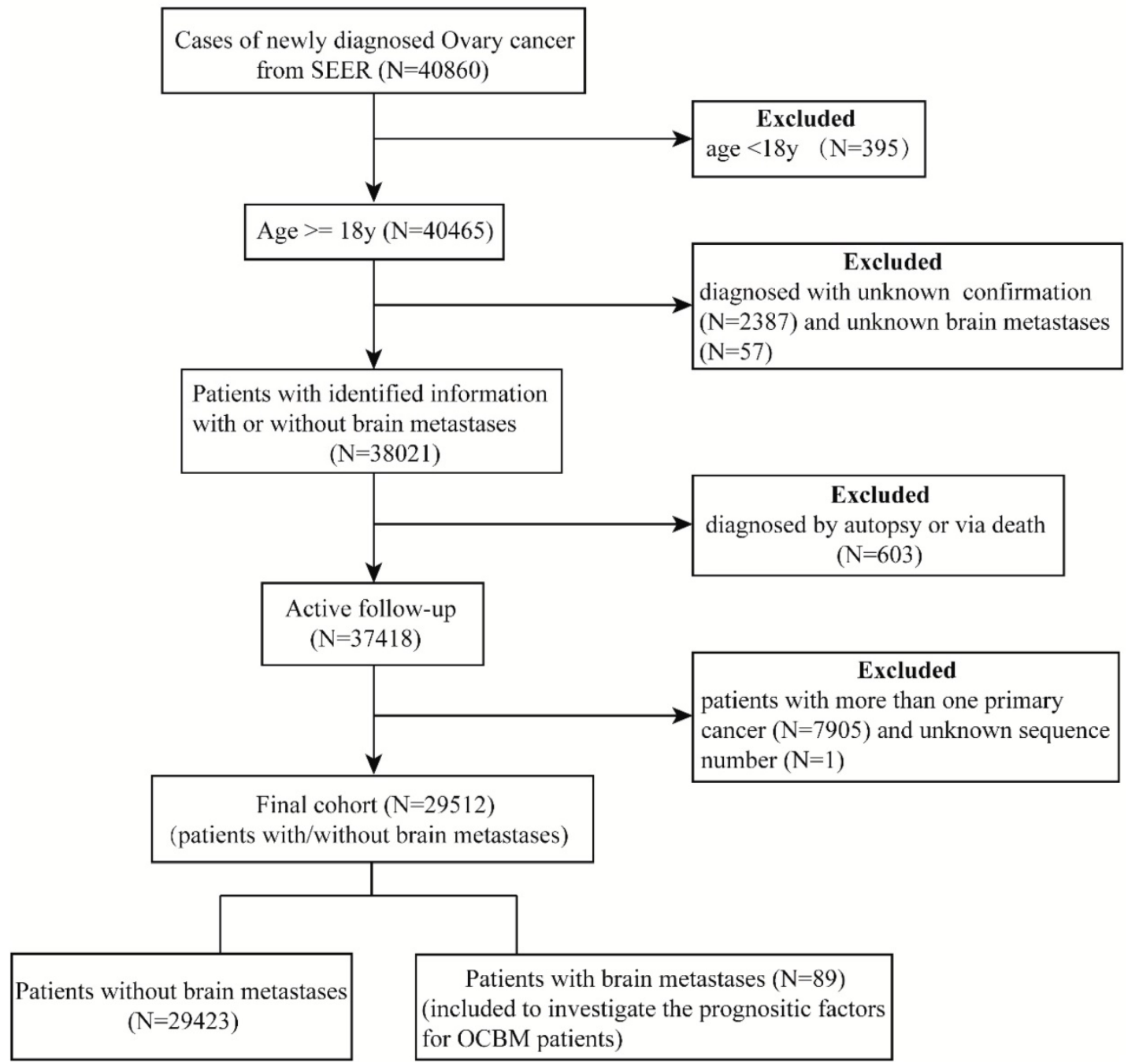

Figure 1. Data extraction flow chart from the SEER database. 
Table 1. Clinical Characteristics of Patients for Ovarian Cancer Patients Diagnosed with and without Brain Metastases

\begin{tabular}{|c|c|c|c|c|c|c|}
\hline \multirow[t]{2}{*}{ Variables } & \multicolumn{3}{|l|}{ Patients, No. } & \multicolumn{2}{|c|}{ Proportion of Brain Metastases, $\%$} & \multirow{2}{*}{$\begin{array}{l}\text { Survival among } \\
\text { Patients with Brain } \\
\text { Metastases, Median } \\
\text { (IQR), mo }\end{array}$} \\
\hline & $\mathrm{OC}(\mathrm{N}=29512)$ & $\begin{array}{l}\text { OC with Brain } \\
\text { Metastases }(\mathrm{N}=89)\end{array}$ & $\begin{array}{l}\text { OC without Brain } \\
\text { Metastases } \\
(\mathrm{N}=29423)\end{array}$ & Among Entire cohort & $\begin{array}{l}\text { Among Metastatic } \\
\text { subset }\end{array}$ & \\
\hline \multicolumn{7}{|l|}{ Age (years) } \\
\hline Median (range) & $62(18-102)$ & & & - & -- & \\
\hline $18-58$ & 12359 & 28 & 12331 & 0.23 & 2.72 & $3(2-11.25)$ \\
\hline$\geq 59$ & 17153 & 61 & 17092 & 0.36 & 2.42 & $2(0-10)$ \\
\hline \multicolumn{7}{|l|}{ Marital status } \\
\hline Married & 14188 & 35 & 14153 & 0.25 & 2.26 & $3(1-12)$ \\
\hline Single & 6565 & 22 & 6542 & 0.34 & 2.77 & $2(0-10.5)$ \\
\hline Divorced & 2990 & 8 & 2982 & 0.27 & 2.18 & $1(0-2)$ \\
\hline Widowed & 4424 & 17 & 4408 & 0.38 & 2.51 & $2(0-7)$ \\
\hline Unknown & 1345 & 7 & 1338 & 0.52 & 4.38 & $4(2-10)$ \\
\hline \multicolumn{7}{|l|}{ Race } \\
\hline White & 23792 & 70 & 23722 & 0.29 & 2.50 & $2(0-9)$ \\
\hline Black & 2692 & 12 & 2692 & 0.45 & 2.64 & $2(0.25-36)$ \\
\hline Others & 2852 & 5 & 2847 & 0.18 & 1.75 & $2(0-18.5)$ \\
\hline Unknown & 176 & 2 & 174 & 1.14 & 22.22 & 10.5 (NA) \\
\hline \multicolumn{7}{|l|}{ Origin recode } \\
\hline Hispanic & 4136 & 11 & 4125 & 0.27 & 2.43 & $6(2-12)$ \\
\hline None-hispanic & 25376 & 78 & 25298 & 0.31 & 2.52 & $2(0-10)$ \\
\hline \multicolumn{7}{|l|}{ Laterality } \\
\hline Unilateral & 15441 & 32 & 15409 & 0.42 & 1.51 & \\
\hline Bilateral & 9362 & 23 & 9339 & 0.25 & 1.24 & $6(2-15)$ \\
\hline Unknown & 4709 & 34 & 4675 & 0.72 & 2.05 & $1.5(0-6.25)$ \\
\hline \multicolumn{7}{|l|}{ Tumor staging } \\
\hline I & 6966 & 0 & 6966 & 0 & 0 & NA- \\
\hline II & 2435 & 0 & 2435 & 0 & 0 & NA \\
\hline III & 10799 & 1 & 10798 & 0.01 & 33.33 & $6(\mathrm{NA})$ \\
\hline IV & 8239 & 87 & 8152 & 1.06 & 2.47 & $2(0-10)$ \\
\hline Unknown & 1073 & 1 & 1072 & 0.09 & 4.76 & 2(NA) \\
\hline \multicolumn{7}{|l|}{$\mathrm{T}$ staging } \\
\hline $\mathrm{T} 1$ & 7811 & 11 & 7800 & 0.14 & 6.55 & $2(0-12)$ \\
\hline $\mathrm{T} 2$ & 3724 & 9 & 3265 & 0.24 & 3.01 & $4(1-12.5)$ \\
\hline $\mathrm{T} 3$ & 15084 & 26 & 15058 & 0.17 & 1.22 & $5(2-12.75)$ \\
\hline Unknown & 2893 & 43 & 2850 & 1.49 & 4.57 & $2(0-6)$ \\
\hline N staging & & & & & & \\
\hline No & 20246 & 45 & 20201 & 0.22 & 2.73 & $2(0-12.5)$ \\
\hline N1 & 6202 & 21 & 6181 & 0.34 & 1.91 & $2(0.5-9)$ \\
\hline Unknown & 3064 & 23 & 3041 & 0.75 & 2.89 & $3(1-7)$ \\
\hline Histology & & & & & & \\
\hline Epithelial neoplasms & 26303 & 67 & 26236 & 0.25 & 2.12 & $2(1-10)$ \\
\hline Gonadal neoplasms & 1297 & 2 & 1295 & 0.15 & 5.00 & 21.5(12-NA) \\
\hline Others & 1071 & 3 & 1068 & 0.28 & 2.07 & $12(2-\mathrm{NA})$ \\
\hline Unknown & 841 & 17 & 824 & 2.02 & 8.37 & $0(0-2.5)$ \\
\hline Treatment $^{\mathrm{a}}$ & & & & & & \\
\hline Others & 3371 & 37 & 3334 & 1.10 & 3.98 & $0(0-2)$ \\
\hline CSR & 213 & 13 & 200 & 6.10 & 29.55 & $12(5.5-39)$ \\
\hline RS & 24 & 3 & 21 & 12.50 & 60.00 & $2(2-N A)$ \\
\hline SC & 17127 & 5 & 17122 & 0.03 & 0.35 & $20(18-56.5)$ \\
\hline $\mathrm{RC}$ & 77 & 15 & 62 & 19.48 & 31.25 & $4(2-10)$ \\
\hline $\mathrm{R}$ & 38 & 6 & 32 & 15.79 & 30.00 & $0.5(0-2.5)$ \\
\hline$S$ & 5647 & 2 & 5645 & 0.04 & 1.18 & $4.5(3-\mathrm{NA})$ \\
\hline $\mathrm{C}$ & 3015 & 8 & 3007 & 0.27 & 0.90 & $3(0.25-7)$ \\
\hline Surgery & & & & & & \\
\hline $\mathrm{U} / \mathrm{BSO}$ & 12146 & 14 & 12132 & 0.12 & 2.77 & $12(7.5-30.75)$ \\
\hline Cytoreductive & 10360 & 9 & 10351 & 0.09 & 0.83 & $12(2-35.5)$ \\
\hline Others & 505 & 0 & 505 & 0 & 0 & NA \\
\hline No & 6501 & 66 & 6435 & 1.02 & 3.50 & $2(0-4)$ \\
\hline Radiotherapy & & & & & & \\
\hline No & 29160 & 52 & 29108 & 0.18 & 1.52 & $1.5(0-6)$ \\
\hline Yes & 352 & 37 & 315 & 10.51 & 31.62 & $4(2-12)$ \\
\hline Chemotherapy & & & & & & \\
\hline No & 9080 & 48 & 9032 & 0.53 & 4.27 & $1(0-2)$ \\
\hline Yes & 20432 & 41 & 20391 & 0.20 & 1.69 & $9(2-16)$ \\
\hline $\begin{array}{l}\text { Radiation Sequence w } \\
\text { surgery }^{b}\end{array}$ & & & & & & \\
\hline RBS & 15 & 1 & 14 & 6.67 & 14.29 & $9(\mathrm{NA})$ \\
\hline RAS & 235 & 17 & 218 & 7.23 & 35.42 & $10(2-14)$ \\
\hline RBAS & 1 & 0 & 1 & 0 & 0 & NA \\
\hline Others & 29261 & 71 & 29190 & 0.24 & 2.03 & $2(0-6)$ \\
\hline
\end{tabular}




\begin{tabular}{|c|c|c|c|c|c|c|}
\hline \multirow[t]{2}{*}{ Variables } & \multicolumn{3}{|l|}{ Patients, No. } & \multicolumn{2}{|c|}{ Proportion of Brain Metastases, $\%$} & \multirow{2}{*}{$\begin{array}{l}\text { Survival among } \\
\text { Patients with Brain } \\
\text { Metastases, Median } \\
\text { (IQR), mo }\end{array}$} \\
\hline & $\mathrm{OC}(\mathrm{N}=\mathbf{2 9 5 1 2})$ & $\begin{array}{l}\text { OC with Brain } \\
\text { Metastases }(\mathrm{N}=89)\end{array}$ & $\begin{array}{l}\text { OC without Brain } \\
\text { Metastases } \\
(\mathrm{N}=29423)\end{array}$ & Among Entire cohort & $\begin{array}{l}\text { Among Metastatic } \\
\text { subset }\end{array}$ & \\
\hline No & 29160 & 61 & 29099 & 0.21 & 1.90 & $2(0-11)$ \\
\hline Yes & 310 & 24 & 286 & 7.74 & 7.74 & $3(0-8.5)$ \\
\hline Unknown & 42 & 4 & 38 & 9.52 & 18.18 & $2.5(0.5-9.75)$ \\
\hline \multicolumn{7}{|l|}{ Liver Met } \\
\hline No & 27249 & 62 & 27187 & 0.23 & 4.47 & $2(0-10.5)$ \\
\hline Yes & 2120 & 21 & 2099 & 0.99 & 0.99 & $2(0.5-5)$ \\
\hline Unknown & 143 & 6 & 137 & 4.20 & 15.00 & $2(0-12.75)$ \\
\hline \multicolumn{7}{|l|}{ Lung Met } \\
\hline No & 27538 & 49 & 27489 & 0.18 & 2.85 & $2(0.5-11)$ \\
\hline Yes & 1773 & 37 & 1736 & 2.09 & 2.09 & $2(0-8)$ \\
\hline Unknown & 201 & 3 & 198 & 1.49 & 5.36 & 0 (NA) \\
\hline \multicolumn{7}{|l|}{ CA125 } \\
\hline Normal & 2654 & 4 & 2650 & 0.15 & 5.13 & $16.5(6-54.75)$ \\
\hline Elevated & 20241 & 49 & 20192 & 0.24 & 1.80 & $2(0-8)$ \\
\hline Unknown & 6617 & 36 & 6581 & 0.54 & 4.85 & $2(0-11.5)$ \\
\hline \multicolumn{7}{|c|}{ Insurance situation } \\
\hline No & 1096 & 7 & 1089 & 0.64 & 4.55 & $2(0-2)$ \\
\hline Yes & 27927 & 77 & 27846 & 0.28 & 2.31 & $2(0-12)$ \\
\hline Unknown & 489 & 5 & 484 & 1.02 & 8.33 & $3(2.5-6.5)$ \\
\hline \multicolumn{7}{|l|}{ Residence type } \\
\hline Rural & 482 & 1 & 481 & 0.21 & 1.67 & 2(NA) \\
\hline Urban & 3376 & 12 & 3364 & 0.36 & 3.10 & $1(0-2.75)$ \\
\hline Metropolitan & 25630 & 76 & 25554 & 0.30 & 2.46 & $2.5(0.25-12)$ \\
\hline Unknown & 24 & 0 & 24 & 0 & 0 & $\mathrm{NA}$ \\
\hline
\end{tabular}

Abbreviations: OC: ovary cancer; IQR: interquartile range; CI: confidence interval.

a including CSR: chemotherapy, surgery, and radiotherapy, RS: radiotherapy and surgery, SC: surgery and chemotherapy, RC: radiotherapy and chemotherapy, R: radiotherapy alone, S: surgery alone, C: chemotherapy alone, others: other treatment except for radiotherapy, surgery, and chemotherapy.

bincluding RBS: radiation before surgery, RAS: radiotherapy after surgery, RBAS: radiotherapy before and after surgery, others: without surgery or radiotherapy or unknown sequence.

According to the univariable logistic regression in the entire cohort (Table S1), fourteen factors achieved significance ( $p$ <0.05), including age, laterality, histology, T, N, surgery, radiotherapy, chemotherapy, radiation sequence with surgery, the presence of bone, lung and liver metastasis, CA125, and insurance type. As is shown in Table 1, the elder age $(0.36 \%)$ was prone to presenting brain metastases than younger age $(0.23 \%)(\mathrm{P}<0.05)$, the unknown laterality $(0.72 \%)$ was more likely to be OCBM than unilateral laterality $(0.42 \%, \mathrm{P}<0.0001)$, the tumor staging grade IV $(1.06 \%)$, and unspecified $(0.09 \%)$ got more risk of OCBM compared to grade III $(0.01 \%$, $\mathrm{P}<0.0001$, respectively), unknown $\mathrm{N}$ staging $(0.75 \%)$ was more likely to develop OCBM than N0 $(0.22 \%$, $\mathrm{P}<0.0001)$ among the entire cohort. For histology, unspecified $(2.02 \%$ and $8.37 \%)$ had a higher proportion of brain metastasis than epithelial neoplasms $(0.25 \%$ and $2.21 \%) \quad(\mathrm{P}<0.0001)$. In the treatment, the absence of chemotherapy $(0.53 \%$ and $4.27 \%$ ) had a significantly higher likelihood of developing brain metastasis than chemotherapy treatment $(0.20 \%$ and $1.69 \%)(\mathrm{P}<0.0001)$. Besides, the brain metastases rate of uninsured patients $(0.64 \%)$ was higher than insured patients $(0.28 \%) \quad(\mathrm{P}<0.001)$ among the entire cohort, which might due to the patients with insurance might suffer less risk to develop metastatic diseases for they could receive more medical intervention.
We then put these factors into multivariable logistic regression and found that laterality, histology, surgery, radiotherapy, chemotherapy, bone met, liver met and lung met had significance among the entire cohort and histology, radiotherapy, chemotherapy, bone met, liver met and lung met achieved significance among the subset with metastatic disease cohort. According to the multivariable logistic regression based on the entire cohort (Table 2), laterality (bilateral vs unilateral, OR, 2.251; 95\%CI, 1.206-4.204; $\mathrm{P}=0.011$ ), unspecified histology (vs epithelia neoplasms; OR, 2.568; 95\%CI, 1.246-5.293; $\mathrm{P}=0.011$ ); surgery no surgery (vs U/BSO; OR, 4.047, 95\%CI, 1.702-9.624; $\mathrm{P}=0.002$ ); radiotherapy (vs no radiotherapy; OR, 32.268; 95\%CI, 15.423-67.508; $\mathrm{P}<0.0001$ ) and chemotherapy (vs no chemotherapy; OR, 0.503; 95\% CI, 0.287-0.881; $\mathrm{P}=0.016$ ), bone met (vs no bone met; OR, 5.095; 95\%CI, 2.737-9.488; P<0.0001) and unknown (vs no bone met; OR, 7.271; 95\%CI, 1.811-29.190; $\mathrm{P}=0.005$ ), liver met (vs no liver met; $\mathrm{OR}$, 1.072; 95\%CI, 0.581-1.977; P>0.05) and unknown (vs no liver met; $\mathrm{OR}, 5.266$; 95\% CI, 1.706-16.250; $\mathrm{P}=0.004)$, lung met (vs no lung met; OR, 5.328; 95\%CI, 3.124-9.085; $\mathrm{P}<0.0001$ ) and unknown (vs no lung met; OR, 1.627; 95\%CI, 0.369-7.176; $\mathrm{P}=0.521)$. The multivariable logistic regression results of the subset with metastatic disease were also presented in Table 2. 
Table 2. Multivariate Logistic Regression for the Patients with Brain Metastases at Diagnosis of Ovary Cancer

\begin{tabular}{|c|c|c|c|c|c|c|c|}
\hline \multirow[t]{2}{*}{ Variables } & \multicolumn{3}{|c|}{ Patients, No } & \multicolumn{2}{|l|}{$\begin{array}{l}\text { Among Entire } \\
\text { Cohort }\end{array}$} & \multicolumn{2}{|c|}{$\begin{array}{l}\text { Among Subset with Metastatic } \\
\text { Disease }\end{array}$} \\
\hline & $\begin{array}{l}\text { Patients } \\
(\mathrm{N}=29512)\end{array}$ & $\begin{array}{l}\text { With Metastatic Disease } \\
(\mathrm{N}=3546)\end{array}$ & $\begin{array}{l}\text { With Brain } \\
\text { Metastases }(\mathrm{N}=89)\end{array}$ & OR $(95 \% \mathrm{CI})$ & $\begin{array}{l}P \\
\text { value }\end{array}$ & OR $(95 \% \mathrm{CI})$ & $P$ value \\
\hline \multicolumn{8}{|l|}{ Age (years) } \\
\hline $18-58$ & 12359 & 1028 & 28 & Reference & & Reference & \\
\hline$\geq 59$ & 17153 & 2518 & 61 & $1.331(0.760-2.329)$ & 0.317 & $0.742(0.412-1.337)$ & 0.321 \\
\hline \multicolumn{8}{|l|}{ Laterality } \\
\hline Unilateral & 15441 & 1192 & 32 & Reference & & Reference & \\
\hline Bilateral & 9362 & 1130 & 23 & $2.251(1.206-4.204)$ & 0.011 & $1.649(0.831-3.270)$ & 0.152 \\
\hline Unknown & 4709 & 1224 & 34 & $0.742(0.403-1.366)$ & 0.338 & $0.564(0.298-1.067)$ & 0.078 \\
\hline \multicolumn{8}{|l|}{ T staging } \\
\hline $\mathrm{T} 1$ & 7811 & 168 & 11 & Reference & & Reference & \\
\hline $\mathrm{T} 2$ & 3724 & 299 & 9 & $0.607(0.225-1.636)$ & 0.324 & $0.466(0.151-1.444)$ & 0.186 \\
\hline $\mathrm{T} 3$ & 15084 & 2138 & 26 & $0.706(0.301-1.657)$ & 0.424 & $0.455(0.179-1.152)$ & 0.097 \\
\hline Unknown & 2893 & 942 & 43 & $1.555(0.645-3.750)$ & 0.325 & $0.826(0.335-2.037)$ & 0.679 \\
\hline \multicolumn{8}{|l|}{$\mathrm{N}$ staging } \\
\hline N0 & 20246 & 1650 & 45 & Reference & & Reference & \\
\hline N1 & 6202 & 1101 & 21 & $0.689(0.371-1.279)$ & 0.238 & $0.837(0.452-1.551)$ & 0.571 \\
\hline Unknown & 3064 & 795 & 23 & $0.678(0.364-1.262)$ & 0.220 & $0.682(0.356-1.306)$ & 0.248 \\
\hline \multicolumn{8}{|l|}{ Histology } \\
\hline Epithelial neoplasms & 26303 & 3158 & 67 & Reference & & Reference & \\
\hline Gonadal neoplasms & 1297 & 40 & 2 & $0.744(0.156-3.540)$ & 0.710 & $2.052(0.333-12.655)$ & 0.439 \\
\hline Others & 1071 & 145 & 3 & $0.762(0.201-2.886)$ & 0.689 & $1.280(0.345-4.754)$ & 0.712 \\
\hline Unknown & 841 & 203 & 17 & $2.406(1.226-4.725)$ & 0.016 & $2.568(1.246-5.293)$ & 0.011 \\
\hline \multicolumn{8}{|l|}{ Surgery } \\
\hline $\mathrm{U} / \mathrm{BSO}$ & 12146 & 506 & 14 & Reference & & Reference & \\
\hline Cytoreductive & 10360 & 1078 & 9 & $0.648(0.253-1.659)$ & 0.368 & $0.500(0.175-1.428)$ & 0.195 \\
\hline Others & 505 & 76 & 0 & $0.000(0.000-\mathrm{NA})$ & 0.993 & $0.000(0.000-\mathrm{NA})$ & 0.997 \\
\hline No & 6501 & 1886 & 66 & $4.047(1.702-9.624)$ & 0.002 & $1.766(0.708-4.404)$ & 0.222 \\
\hline \multicolumn{8}{|l|}{ Radiotherapy } \\
\hline No & 29160 & 117 & 52 & Reference & & Reference & \\
\hline Yes & 352 & 3429 & 37 & 32.268 (15.423-67.508) & $<0.0001$ & $27.036(12.397-58.963)$ & $<0.0001$ \\
\hline \multicolumn{8}{|l|}{ Chemotherapy } \\
\hline No & 9080 & 2421 & 48 & Reference & & Reference & \\
\hline Yes & 20432 & 1125 & 41 & $0.503(0.287-0.881)$ & 0.016 & $0.432(0.236-0.791)$ & 0.006 \\
\hline \multicolumn{8}{|c|}{$\begin{array}{l}\text { Radiation Sequence with } \\
\text { surgery }\end{array}$} \\
\hline RBS & 15 & 7 & 1 & Reference & & Reference & \\
\hline RAS & 235 & 48 & 17 & $2.496(0.259-24.077)$ & 0.429 & $1.576(0.141-17.601)$ & 0.712 \\
\hline RBAS & 1 & 1 & 0 & $0.000(0.000-\mathrm{NA})$ & 1.000 & $0.000(0.000-\mathrm{NA})$ & 1.000 \\
\hline Others & 29261 & 3490 & 71 & $0.564(0.057-5.593)$ & 0.625 & $0.994(0.089-11.057)$ & 0.996 \\
\hline \multicolumn{8}{|l|}{ Bone Met } \\
\hline No & 29160 & 310 & 61 & Reference & & Reference & \\
\hline Yes & 310 & 3214 & 24 & $5.095(2.737-9.488)$ & $<0.0001$ & $0.706(0.350-1.424)$ & 0.331 \\
\hline Unknown & 42 & 22 & 4 & 7.271(1.811-29.190) & 0.005 & $9.748(2.073-45.845)$ & 0.004 \\
\hline \multicolumn{8}{|l|}{ Liver Met } \\
\hline No & 27249 & 2120 & 62 & Reference & & Reference & \\
\hline Yes & 2120 & 1386 & 21 & $1.072(0.581-1.977)$ & 0.824 & $0.144(0.073-0.281)$ & $<0.0001$ \\
\hline Unknown & 143 & 40 & 6 & $5.266(1.706-16.250)$ & 0.004 & $3.836(1.104-13.323)$ & 0.034 \\
\hline \multicolumn{8}{|l|}{ Lung Met } \\
\hline No & 27538 & 1773 & 49 & Reference & & Reference & \\
\hline Yes & 1773 & 1717 & 37 & $5.328(3.124-9.085)$ & $<0.0001$ & $0.339(0.178-0.643)$ & 0.001 \\
\hline Unknown & 201 & 56 & 3 & $1.627(0.369-7.176)$ & 0.521 & $0.700(0.110-4.464)$ & 0.706 \\
\hline CA125 & & & & & & & \\
\hline Normal & 2654 & 78 & 4 & Reference & & Reference & \\
\hline Elevated & 20241 & 2726 & 49 & $1.437(0.439-4.712)$ & 0.549 & $0.957(0.251-3.647)$ & 0.949 \\
\hline Unknown & 6617 & 742 & 36 & $2.234(0.674-7.400)$ & 0.188 & $1.507(0.390-5.823)$ & 0.552 \\
\hline Insurance situation & & & & & & & \\
\hline No & 1096 & 3332 & 7 & Reference & & Reference & \\
\hline Yes & 27927 & 154 & 77 & $0.506(0.201-1.274)$ & 0.148 & $0.674(0.250-1.816)$ & 0.436 \\
\hline Unknown & 489 & 60 & 5 & $1.751(0.473-6.484)$ & 0.402 & 1.721(0.379-7.817) & 0.482 \\
\hline
\end{tabular}

Abbreviations:

OR: odds ratio; CI: confidence interval.

\section{Survival}

The univariate analysis of all-cause mortality among the entire cohort as well as the subset with brain metastases were presented in Table S2. Seven factors were significantly associated with overall survival among brain metastases subset $(\mathrm{p}<0.05)$. They were marital status, histology, treatment, surgery, radiotherapy, chemotherapy, and insurance situation. On Cox regression analysis among brain metastases in the model 1 , the results (Table 3 ) 
showed that divorced (vs married; HR, 2.688, 95\% CI, 1.102-6.560; $\mathrm{P}=0.030$ ), no surgery (vs $\mathrm{U} / \mathrm{BSO} ; \mathrm{HR}$, $3.712,95 \% \mathrm{CI}, 1.519-9.075$; $\mathrm{P}=0.004$ ) were significantly associated with increased all-cause mortality and chemotherapy (vs no chemotherapy; HR, 0.341, 95\% CI, 0.169-0.687; $\mathrm{P}=0.003$ ) reduced the risk of death. While single marital status (vs married; HR, 0.679, 95\% CI, 0.351-1.313; $\mathrm{P}=0.249)$ and cytoreductive surgery (vs U/BSO; $\mathrm{HR}, 1.042,95 \% \mathrm{CI}, 0.317-3.421$; $\mathrm{P}>0.05$ ) didn't achieve any statistical difference in OS in model 1. Moreover, marital status and treatment significantly correlated with all-cause mortality in model 2 (Table 3). Marital status divorced (vs married; $\mathrm{HR}, 2.672,95 \% \mathrm{CI}, 1.027-6.953 ; \mathrm{P}=0.044)$ and treatment were significantly associated with increased all-cause mortality. Intriguingly, being divorced was associated with a higher risk of mortality than married status, while single (vs married; HR, 0.643, 95\%CI, 0.327-1.264; $\mathrm{P}=0.200$ ) was not statistical significant. And treatment chemotherapy+surgery+radiotherapy (vs others; HR, 0.096, 95\% CI, 0.030-0.308; P<0.0001), surgery plus chemotherapy (vs others; HR, 0.081,
95\%CI, 0.021-0.312; $\mathrm{P}<0.0001)$, radiotherapy plus chemotherapy (vs others; HR, 0.260, 95\% CI, $0.115-0.587 ; \mathrm{P}=0.001)$ were found to be protective interventions of survival in model 2. Generally, it seemed that divorced marital status, absence of positive treatment including any combination with surgery, chemotherapy, and radiotherapy had increased risk of mortality among OCBM patients.

The median survival time of the OCBM cohort was 2.0 months (IQR: 0.0-10.0mo) (Table 1, Figure 2A). The median survival time for the patients with unilateral/bilateral (salpingo-) oophorectomy was 12.0 months (7.5-30.75mo), with cytoreductive surgery was 12.0 months (IQR: 2.0-35.5 mo), without surgery was 2.0 months (IQR: 0.0-4.0 mo) (Figure 3A). Median survival time of patients receiving chemotherapy was 9.0 months (IQR: 2.0-16.0 mo), while no chemotherapy was 1.0 month (IQR: $0.0-2.0 \mathrm{mo}$ ) (Figure 3B). The median survival time for radiation-treated patients was 4.0 months (IQR: 2.0-12.0mo), among those without radiation was 1.5 months (IQR: 0.0-6.0 mo) (Figure 3C).

Table 3. Multivariate Cox Regression for the Ovary Cancer Patients with Brain Metastases

\begin{tabular}{|c|c|c|c|c|c|c|}
\hline \multirow[t]{2}{*}{ Variables } & \multicolumn{2}{|l|}{ Patients, No. } & \multicolumn{2}{|c|}{ All-Cause Mortality (model 1) } & \multicolumn{2}{|c|}{ All-Cause Mortality (model 2) } \\
\hline & Patients (N=29512) & With Brain Metastases $(\mathrm{N}=89)$ & HR $(95 \% C I)$ & Pvalue & HR $(95 \% \mathrm{CI})$ & Pvalue \\
\hline \multicolumn{7}{|l|}{ Marital status } \\
\hline Married & 14188 & 35 & Reference & & Reference & \\
\hline Single & 6565 & 22 & $0.679(0.351-1.313)$ & 0.249 & $0.643(0.327-1.264)$ & 0.200 \\
\hline Divorced & 2990 & 8 & $2.688(1.102-6.560)$ & 0.030 & $2.672(1.027-6.953)$ & 0.044 \\
\hline Widowed & 4424 & 17 & $0.710(0.355-1.419)$ & 0.332 & $0.659(0.324-1.341)$ & 0.250 \\
\hline Unknown & 1345 & 7 & $0.515(0.185-1.429)$ & 0.202 & $0.485(0.170-1.384)$ & 0.176 \\
\hline \multicolumn{7}{|l|}{ Histology } \\
\hline Epithelial neoplasms & 26303 & 67 & Reference & & Reference & \\
\hline Gonadal neoplasms & 1297 & 2 & $0.215(0.028-1.670)$ & 0.142 & $0.215(0.028-1.675)$ & 0.142 \\
\hline Others & 1071 & 3 & $1.183(0.144-9.737)$ & 0.876 & $0.998(0.105-9.486)$ & 0.999 \\
\hline Unknown & 841 & 17 & $0.921(0.480-1.766)$ & 0.804 & $0.906(0.465-1.767)$ & 0.906 \\
\hline \multicolumn{7}{|l|}{ Treatment } \\
\hline Others & 3371 & 37 & NA & NA & Reference & NA \\
\hline CSR & 213 & 13 & NA & NA & $0.096(0.030-0.308)$ & $<0.0001$ \\
\hline RS & 24 & 3 & NA & NA & $0.283(0.060-1.342)$ & 0.112 \\
\hline SC & 17127 & 5 & NA & NA & $0.081(0.021-0.312)$ & $<0.0001$ \\
\hline $\mathrm{RC}$ & 77 & 15 & NA & NA & $0.260(0.115-0.587)$ & 0.001 \\
\hline $\mathrm{R}$ & 38 & 6 & NA & NA & $1.015(0.357-2.886)$ & 0.978 \\
\hline$S$ & 5647 & 2 & NA & NA & $0.207(0.040-1.086)$ & 0.063 \\
\hline C & 3015 & 8 & NA & NA & $0.428(0.167-1.101)$ & 0.078 \\
\hline \multicolumn{7}{|l|}{ Surgery } \\
\hline $\mathrm{U} / \mathrm{BSO}$ & 12146 & 14 & Reference & & NA & NA \\
\hline Cytoreductive & 10360 & 9 & $1.042(0.317-3.421)$ & 0.946 & NA & NA \\
\hline Others & 505 & 0 & NA & NA & NA & NA \\
\hline No & 6501 & 66 & 3.712(1.519-9.075) & 0.004 & NA & NA \\
\hline Radiotherapy & & & & & NA & NA \\
\hline No & 29160 & 52 & Reference & & NA & NA \\
\hline Yes & 352 & 37 & $0.893(0.481-1.657)$ & 0.720 & NA & NA \\
\hline \multicolumn{7}{|l|}{ Chemotherapy } \\
\hline No & 9080 & 48 & Reference & & NA & NA \\
\hline Yes & 20432 & 41 & $0.341(0.169-0.687)$ & 0.003 & NA & NA \\
\hline \multicolumn{7}{|l|}{ Insurance situation } \\
\hline No & 1096 & 7 & Reference & & Reference & \\
\hline Yes & 27927 & 77 & $0.776(0.315-1.912)$ & 0.582 & $0.754(0.281-2.020)$ & 0.574 \\
\hline Unknown & 489 & 5 & $0.965(0.255-3.655)$ & 0.958 & $0.984(0.242-4.006)$ & 0.984 \\
\hline
\end{tabular}


A
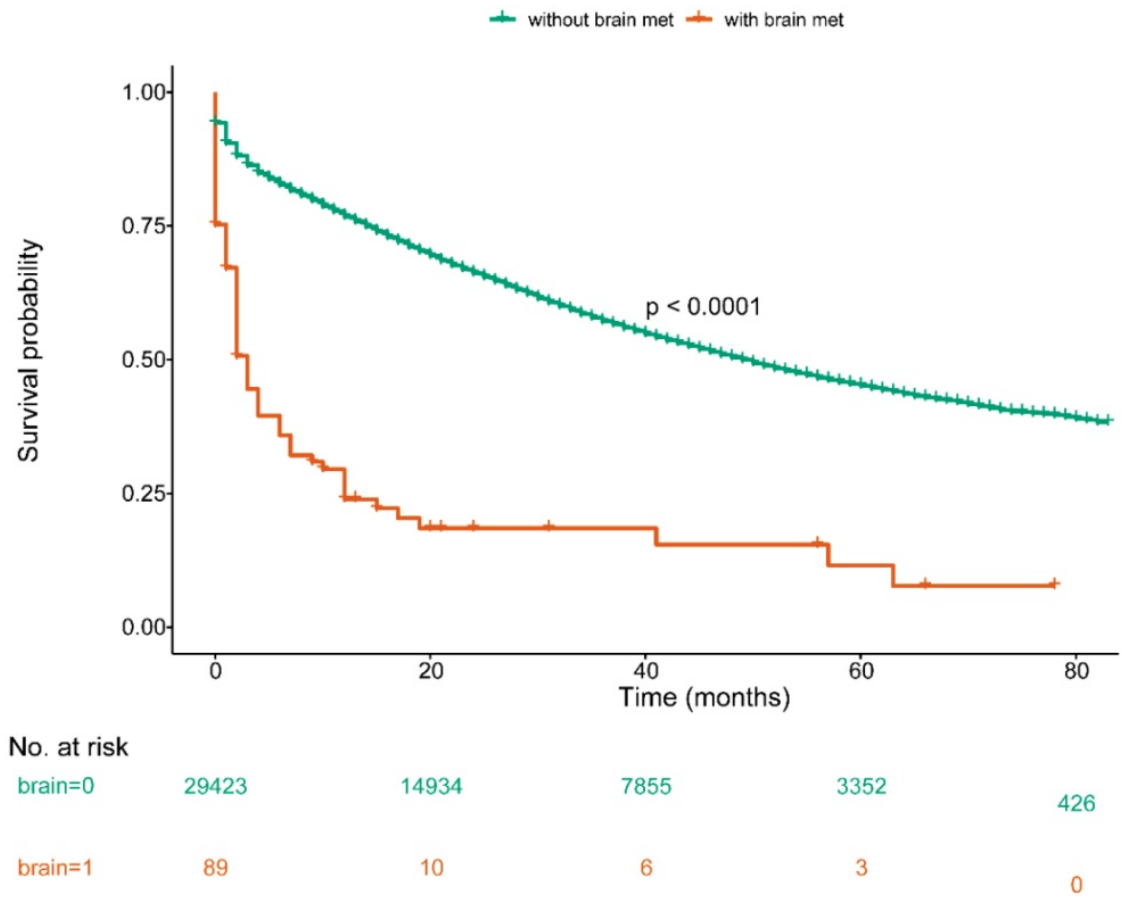

B

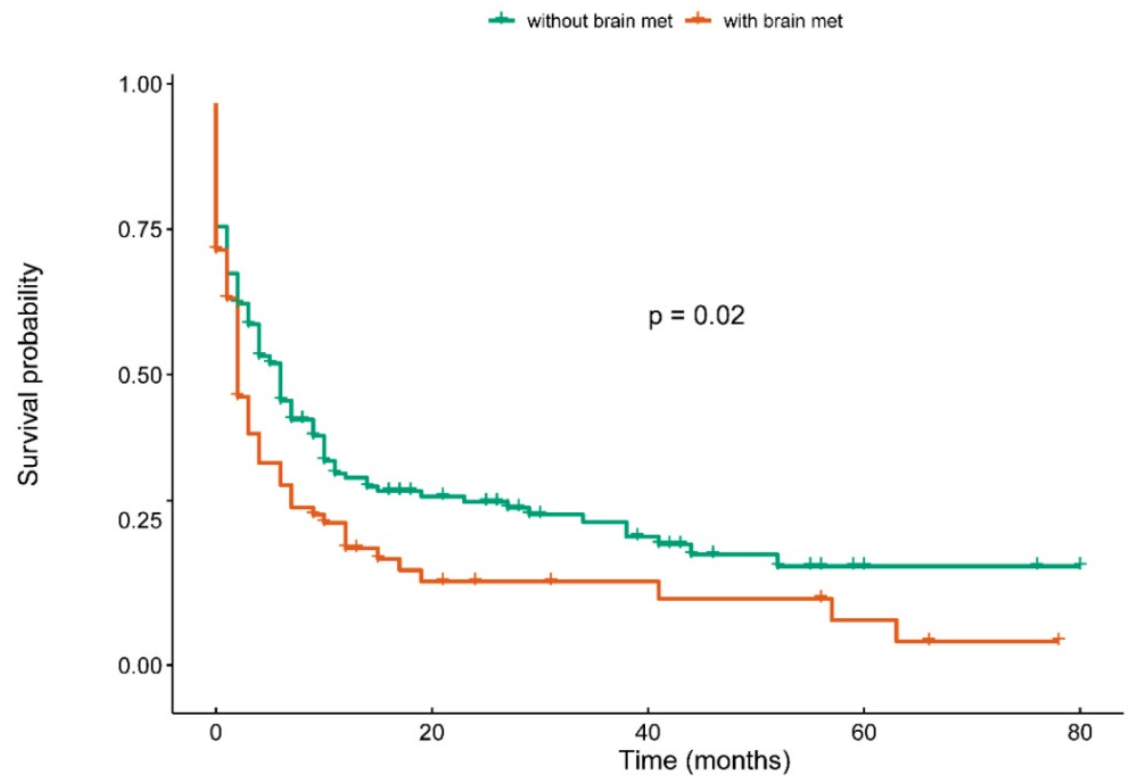

No. at risk

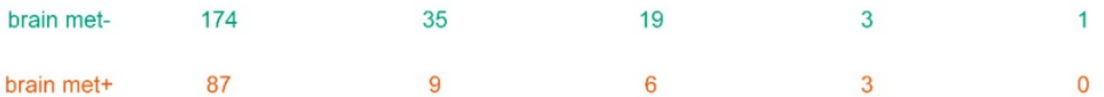

Figure 2. Overall survival among patients with or without de novo brain metastases at ovary cancer diagnosis before and after PSM (A. OS among OC patients with or without BM before PSM; B. OS among OC patients with or without BM after PSM).

Median survival time among the sequence of radiotherapy and surgery indicated those received radiotherapies before surgery were 9.0 months (NA mo), radiation after surgery was 10.0 months (IQR: 2.0-14.0 mo), others were 2.0 months (IQR: 0.0-6.0 mo) (Figure 3D). Median survival time of CSR-treated patients was 12.0 months (IQR: 5.5-39.0mo), RS-treated was 2.0 months (IQR: 2.0-NA mo), SC-treated was 20.0 months (IQR:18.0-56.5mo), RC-treated was 4.0 months (IQR: 2.0-10.0mo), R-treated was 0.5 months (IQR: 0.0-2.5mo), S-treated was 4.5 months (IQR: 3.0-NAmo), C-treated was 3.0 
months (IQR: 0.25-7.0mo), others was 0.0 month (IQR:0.0-2.0mo) (Figure 4). Survival analysis among the number of extracranial metastases was displayed in Figure S1 as supplementary.

\section{Survival analysis among PSM-matched cohort}

Given the confounding factors between groups of with or without brain metastases, a 1:1 ratio PSM was used to balance the baseline clinical characteristics. There was no variable that achieved significant difference between case and controls (Table 4) after matching. In the matched cohort, we included 178 OC patients: 89 cases of brain met group and another 89 cases of without brain met group. Based upon the comparable variables between two groups, we conducted univariate Cox regression to draw a more accurate conclusion: ovary cancer with brain metastases contributed to poor prognosis for

A

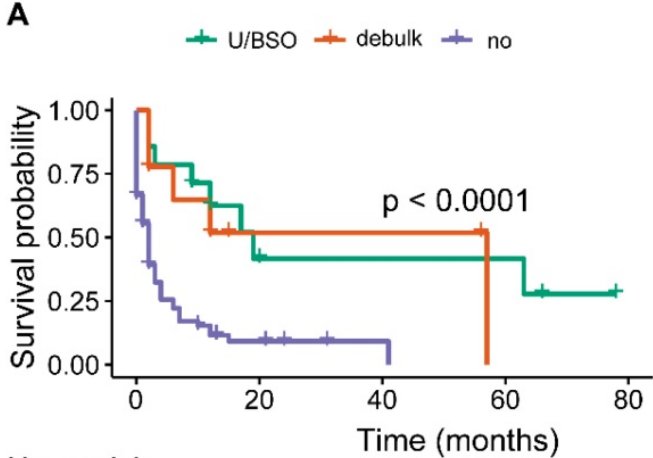

No. at risk U/BSO 14 debulk 9 no $\quad 66$

C

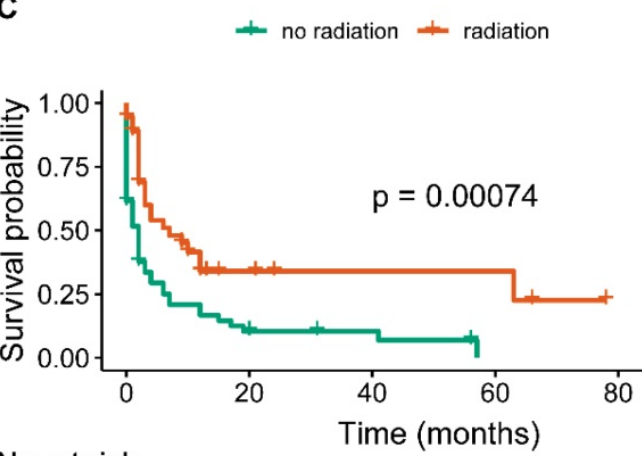

No. at risk no radiation 52

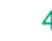

2

4 5
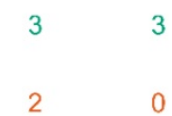

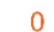

0

0

0
B

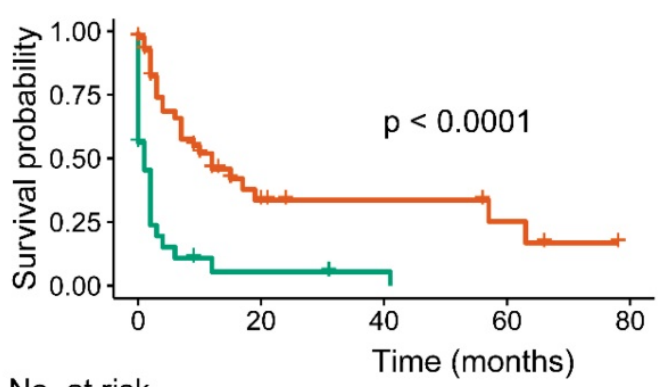

No. at risk

0 no chemo 48

chemo 41

D

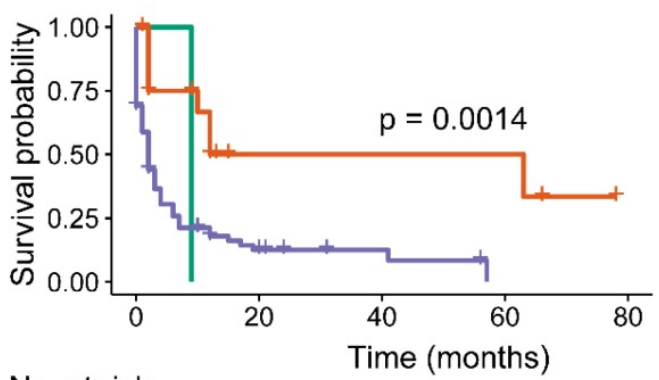

No. at risk

$\begin{array}{ccccc}\text { RBS } 1 & 0 & 0 & 0 & 0 \\ \text { RAS 17 } & 3 & 3 & 3 & 0 \\ \text { others } 71 & 7 & 3 & 0 & 0\end{array}$

Figure 3. Overall survival among patients with OCBM at diagnosis (A, stratified by surgery, B, stratified by chemotherapy; C, stratified by radiation; D, stratified by sequence between surgery and radiation). 


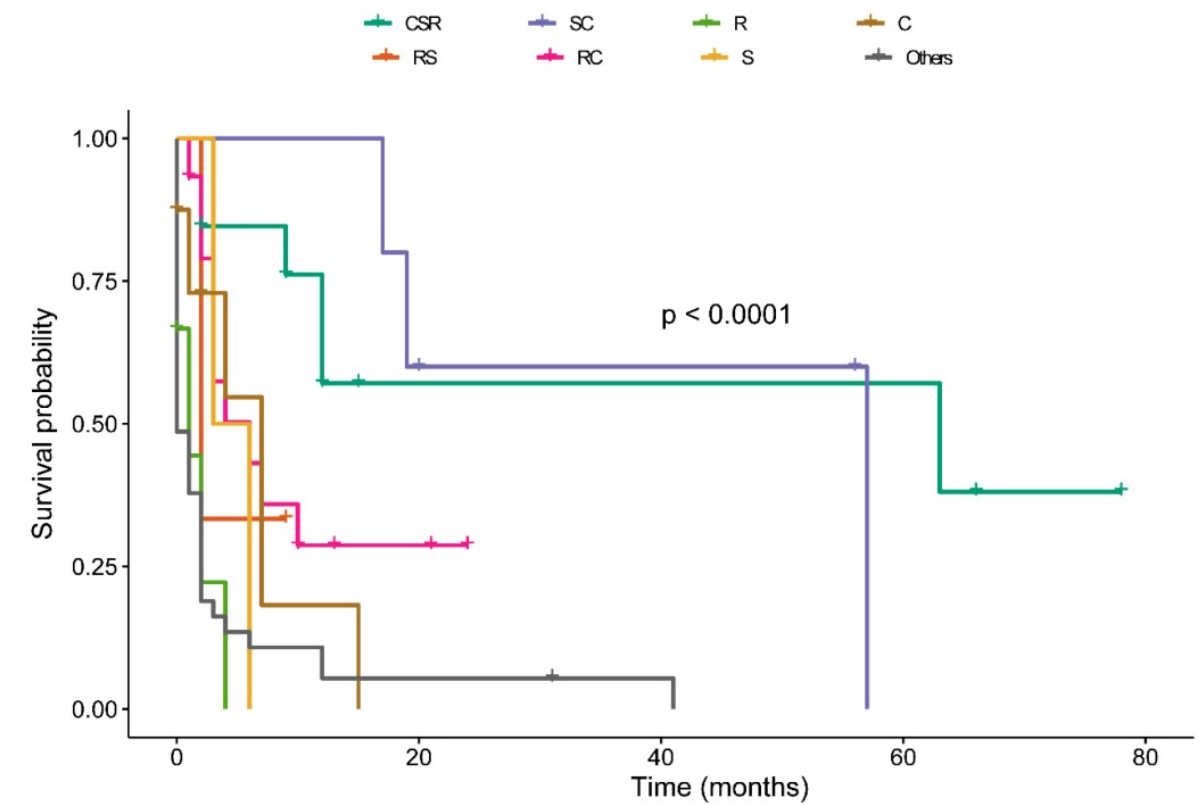

No. at risk

$\begin{array}{llllll}\text { CSR } & 13 & 3 & 3 & 3 & 0 \\ \text { RS } & 3 & 0 & 0 & 0 & 0 \\ \text { SC } & 5 & 3 & 2 & 0 & 0 \\ \text { RC } & 15 & 2 & 0 & 0 & 0 \\ \text { R } & 6 & 0 & 0 & 0 & 0 \\ \text { S } & 2 & 0 & 0 & 0 & 0 \\ \text { C } & 8 & 0 & 0 & 0 & 0 \\ \text { Others } & 37 & 2 & 1 & 0\end{array}$

Figure 4. Overall survival among patients with OCBM at diagnosis stratified by treatment.

Table 4. Baseline Characteristics of Patients for Ovarian Cancer Patients Diagnosed with and without Brain Metastases before and after PSM

\begin{tabular}{|c|c|c|c|c|c|c|}
\hline \multirow[t]{2}{*}{ Variables } & \multicolumn{3}{|l|}{ Before matching } & \multicolumn{3}{|l|}{ After matching } \\
\hline & OC without Brain Metastases ( $N=29423, \%)$ & $\begin{array}{l}\text { OC with Brain } \\
\text { Metastases (N=89, } \\
\%)\end{array}$ & $\begin{array}{l}P \\
\text { value }\end{array}$ & OC without Brain Metastases $(\mathrm{N}=89, \%)$ & $\begin{array}{l}\text { OC with Brain } \\
\text { Metastases }(\mathrm{N}=89, \%)\end{array}$ & $\begin{array}{l}P \\
\text { value }\end{array}$ \\
\hline Age (years) & & & 0.072 & & & 0.884 \\
\hline Median (SD) & $61.26(15.20)$ & $64.16(13.81)$ & & $63.81(17.83)$ & $64.16(13.81)$ & \\
\hline Marital status & & & 0.295 & & & 0.554 \\
\hline Married & $14153(48.1)$ & $35(39.3)$ & & $35(39.3)$ & $35(39.3)$ & \\
\hline Single & $6542(22.2)$ & $22(24.7)$ & & $24(27.0)$ & $22(24.7)$ & \\
\hline Divorced & $2982(10.1)$ & $8(9.0)$ & & $5(5.6)$ & $8(9.0)$ & \\
\hline Widowed & $4408(15.0)$ & $17(19.1)$ & & $22(24.7)$ & $17(19.1)$ & \\
\hline Unknown & $1338(4.5)$ & $7(7.9)$ & & $3(3.4)$ & $7(7.9)$ & \\
\hline Race & & & 0.058 & & & 0.826 \\
\hline White & $23722(80.6)$ & $70(78.7)$ & & $73(82.0)$ & $70(78.7)$ & \\
\hline Black & $2680(9.1)$ & $12(13.5)$ & & $12(13.5)$ & $12(13.5)$ & \\
\hline Others & $2847(9.7)$ & $5(5.6)$ & & $3(3.4)$ & $5(5.6)$ & \\
\hline Unknown & $174(0.6)$ & $2(2.2)$ & & $1(1.1)$ & $2(2.2)$ & \\
\hline Origin recode & & & 0.766 & & & 0.826 \\
\hline None-hispanic & $25298(86.0)$ & $78(87.6)$ & & $76(85.4)$ & $78(87.6)$ & \\
\hline Laterality & & & $<0.001$ & & & 0.405 \\
\hline Unilateral & 15409 (52.4) & $32(36.0)$ & & $30(33.7)$ & $32(36.0)$ & \\
\hline
\end{tabular}




\begin{tabular}{|c|c|c|c|c|c|c|}
\hline \multirow[t]{2}{*}{ Variables } & \multicolumn{3}{|c|}{ Before matching } & \multicolumn{3}{|l|}{ After matching } \\
\hline & OC without Brain Metastases (N=29423, \%) & $\begin{array}{l}\text { OC with Brain } \\
\text { Metastases (N=89, } \\
\%)\end{array}$ & $\begin{array}{l}P \\
\text { value }\end{array}$ & OC without Brain Metastases $(\mathrm{N}=89, \%)$ & $\begin{array}{l}\text { OC with Brain } \\
\text { Metastases }(\mathrm{N}=89, \%)\end{array}$ & $\begin{array}{l}P \\
\text { value }\end{array}$ \\
\hline Bilateral & 9339 (31.7) & $23(25.8)$ & & $17(19.1)$ & $23(25.8)$ & \\
\hline Unknown & 4675 (15.9) & $34(38.2)$ & & $42(47.2)$ & $34(38.2)$ & \\
\hline T staging & & & $<0.001$ & & & 0.592 \\
\hline $\mathrm{T} 1$ & $7800(26.5)$ & $11(12.4)$ & & $7(7.9)$ & $11(12.4)$ & \\
\hline $\mathrm{T} 2$ & $3715(12.6)$ & $9(10.1)$ & & $11(12.4)$ & $9(10.1)$ & \\
\hline T3 & $15058(51.2)$ & $26(29.2)$ & & $32(36.0)$ & $26(29.2)$ & \\
\hline Unknown & $2850(9.7)$ & $43(48.3)$ & & $39(43.8)$ & $43(48.3)$ & \\
\hline N staging & & & $<0.001$ & & & 0.849 \\
\hline No & $20201(68.7)$ & $45(50.6)$ & & $48(53.9)$ & $45(50.6)$ & \\
\hline N1 & $6181(21.0)$ & $21(23.6)$ & & $18(20.2)$ & $21(23.6)$ & \\
\hline Unknown & 3041 (10.3) & $23(25.8)$ & & $23(25.8)$ & $23(25.8)$ & \\
\hline Histology & & & $<0.001$ & & & 0.849 \\
\hline Epithelial neoplasms & $26236(89.2)$ & $67(75.3)$ & & $60(67.4)$ & $67(75.3)$ & \\
\hline Gonadal neoplasms & $1295(4.4)$ & $2(2.2)$ & & $4(4.5)$ & $2(2.2)$ & \\
\hline Others & $1068(3.6)$ & $3(3.4)$ & & $10(11.2)$ & $3(3.4)$ & \\
\hline Unknown & $824(2.8)$ & $17(19.1)$ & & $15(16.9)$ & $17(19.1)$ & \\
\hline Surgery & & & $<0.001$ & & & 0.176 \\
\hline $\mathrm{U} / \mathrm{BSO}$ & $12132(41.2)$ & $14(15.7)$ & & $10(11.2)$ & $14(15.7)$ & \\
\hline Cytoreductive & $10351(35.2)$ & $9(10.1)$ & & 13 (14.6) & $9(10.1)$ & \\
\hline Others & $505(1.7)$ & $0(0.0)$ & & $2(2.2)$ & $0(0.0)$ & \\
\hline No & 6435 (21.9) & $66(74.2)$ & & $64(71.9)$ & $66(74.2)$ & \\
\hline Radiotherapy & & & & & & 1.000 \\
\hline & $315(1.1)$ & 37 (41.6) & $<0.001$ & 37 (41.6) & 37 (41.6) & \\
\hline Chemotherapy & & & & & & 0.653 \\
\hline & 20391 (69.3) & $41(46.1)$ & $<0.001$ & $45(50.6)$ & $41(46.1)$ & \\
\hline $\begin{array}{l}\text { Radiation Sequence } \\
\text { with surgery }\end{array}$ & & & $<0.001$ & & & 0.559 \\
\hline RBS & $14(0.0)$ & $1(1.1)$ & & $1(1.1)$ & $1(1.1)$ & \\
\hline RAS & $218(0.7)$ & $17(19.1)$ & & $23(25.8)$ & $17(19.1)$ & \\
\hline RBAS & $1(0.0)$ & 0 & & 0 & 0 & \\
\hline Others & $29190(99.2)$ & $71(79.8)$ & & $65(73.0)$ & $71(79.8)$ & \\
\hline Treatment & & & $<0.001$ & & & 0.469 \\
\hline CSR & $200(0.7)$ & $13(14.6)$ & & $18(20.2)$ & 13 (14.6) & \\
\hline RS & $21(0.1)$ & $3(3.4)$ & & $2(2.2)$ & $3(3.4)$ & \\
\hline SC & $17122(58.2)$ & $5(5.6)$ & & $1(1.1)$ & $5(5.6)$ & \\
\hline $\mathrm{RC}$ & $62(0.2)$ & $15(16.9)$ & & $12(13.5)$ & $15(16.9)$ & \\
\hline $\mathrm{R}$ & $32(0.1)$ & $6(6.7)$ & & $5(5.6)$ & $6(6.7)$ & \\
\hline S & 5645 (19.2) & $2(2.2)$ & & $4(4.5)$ & $2(2.2)$ & \\
\hline $\mathrm{C}$ & 3007 (10.2) & $8(9.0)$ & & 14 (15.7) & $8(9.0)$ & \\
\hline Others & 3334 (11.3) & 37 (41.6) & & $33(37.1)$ & 37 (41.6) & \\
\hline Bone Met & & & $<0.001$ & & & 0.186 \\
\hline No & 29099 (98.9) & $61(68.5)$ & & $70(78.7)$ & $61(68.5)$ & \\
\hline Yes & $286(1.0)$ & $24(27.0)$ & & 14 (15.7) & $24(27.0)$ & \\
\hline Unknown & $38(0.1)$ & $4(4.5)$ & & $5(5.6)$ & $4(4.5)$ & \\
\hline Liver Met & & & $<0.001$ & & & 0.781 \\
\hline No & 27187 (92.4) & $62(69.7)$ & & $65(73.0)$ & $62(69.7)$ & \\
\hline Yes & $2099(7.1)$ & $21(23.6)$ & & $20(22.5)$ & $21(23.6)$ & \\
\hline Unknown & $137(0.5)$ & $6(6.7)$ & & $4(4.5)$ & $6(6.7)$ & \\
\hline Lung Met & & & $<0.001$ & & & 0.298 \\
\hline No & 27489 (93.4) & $49(55.1)$ & & $52(58.4)$ & $49(55.1)$ & \\
\hline Yes & $1736(5.9)$ & 37 (41.6) & & $30(33.7)$ & 37 (41.6) & \\
\hline Unknown & $198(0.7)$ & $3(3.4)$ & & $7(7.9)$ & $3(3.4)$ & \\
\hline CA125 & & & $<0.001$ & & & 0.127 \\
\hline Normal & $2650(9.0)$ & $4(4.5)$ & & $0(0.0)$ & $4(4.5)$ & \\
\hline Elevated & $20192(68.6)$ & $49(55.1)$ & & $50(56.2)$ & 49 (55.1) & \\
\hline Unknown & $6581(22.4)$ & $36(40.4)$ & & $39(43.8)$ & $36(40.4)$ & \\
\hline Insurance situation & & & 0.001 & & & 0.167 \\
\hline No & $1089(3.7)$ & $7(7.9)$ & & $2(2.2)$ & $7(7.9)$ & \\
\hline Yes & $27850(94.7)$ & $77(86.5)$ & & $84(94.4)$ & $77(86.5)$ & \\
\hline Unknown & $484(1.6)$ & $5(5.6)$ & & $3(3.4)$ & $5(5.6)$ & \\
\hline Residence type & & & 0.905 & & & 0.819 \\
\hline Rural & 481 (1.6) & $1(1.1)$ & & $2(2.2)$ & $1(1.1)$ & \\
\hline Urban & $3364(11.4)$ & $12(13.5)$ & & 13 (14.6) & $12(13.5)$ & \\
\hline Metropolitan & $25554(86.9)$ & $76(85.4)$ & & $74(83.1)$ & $76(85.4)$ & \\
\hline Unknown & $24(0.1)$ & $0(0.0)$ & & $0(0.0)$ & $0(0.0)$ & \\
\hline
\end{tabular}

Abbreviations:

PSM: Propensity Score Matching; SD: Standard Deviation 
Table 5. Univariate and multivariate Cox Regression for the Brain Metastases of Ovary Cancer after PSM.

\begin{tabular}{|c|c|c|c|c|c|c|}
\hline \multirow[t]{2}{*}{ Variables } & \multicolumn{2}{|l|}{ Univariate } & \multicolumn{2}{|l|}{ Multivariate in model 1} & \multicolumn{2}{|c|}{ Multivariate in model 2} \\
\hline & HR (95\%CI) & Pvalue & HR $(95 \% C I)$ & Pvalue & HR $(95 \% \mathrm{CI})$ & Pvalue \\
\hline \multicolumn{7}{|l|}{ Age (years) } \\
\hline $18-58$ & Reference & & Reference & & Reference & \\
\hline$\geq 59$ & $1.575(1.086-2.283)$ & 0.017 & $0.719(0.418-1.237)$ & 0.233 & $0.752(0.435-1.298)$ & 0.306 \\
\hline \multicolumn{7}{|l|}{ Marital status } \\
\hline Married & Reference & & Reference & & Reference & \\
\hline Single & $0.762(0.470-1.233)$ & 0.268 & $0.599(0.344-1.043)$ & 0.070 & $0.548(0.317-0.948)$ & 0.031 \\
\hline Divorced & $2.163(1.201-3.894)$ & 0.010 & $1.977(0.977-4.003)$ & 0.058 & $1.882(0.890-3.981)$ & 0.098 \\
\hline Widowed & $1.665(1.081-2.563)$ & 0.021 & $1.073(0.629-1.829)$ & 0.797 & $1.002(0.583-1.723)$ & 0.994 \\
\hline Unknown & $0.976(0.476-1.999)$ & 0.946 & $0.567(0.238-1.347)$ & 0.199 & $0.532(0.220-1.288)$ & 0.162 \\
\hline \multicolumn{7}{|l|}{ Race } \\
\hline White & Reference & & NA & NA & NA & NA \\
\hline Black & $0.829(0.513-1.340)$ & 0.443 & NA & NA & NA & NA \\
\hline Others & $1.470(0.766-2.820)$ & 0.247 & NA & NA & NA & NA \\
\hline Unknown & $0.466(0.065-3.348)$ & 0.448 & NA & NA & NA & NA \\
\hline \multicolumn{7}{|l|}{ Origin recode } \\
\hline Hispanic & Reference & & Reference & & Reference & NA \\
\hline Non-hispanic & $1.535(0.805-2.928)$ & 0.193 & $0.798(0.369-1.727)$ & 0.298 & $0.923(0.428-1.990)$ & 0.837 \\
\hline \multicolumn{7}{|l|}{ Laterality } \\
\hline Unilateral & Reference & & Reference & & Reference & NA \\
\hline Bilateral & $0.777(0.472-1.278)$ & 0.320 & $0.694(0.379-1.270)$ & 0.236 & $0.743(0.401-1.378)$ & 0.346 \\
\hline Unknown & $1.153(0.788-1.687)$ & 0.463 & $1.119(0.679-1.846)$ & 0.659 & $1.112(0.673-1.837)$ & 0.678 \\
\hline \multicolumn{7}{|l|}{ Tumor staging } \\
\hline I & Reference & & NA & NA & NA & NA \\
\hline II & 0.904(0.000-2.159E67) & 0.999 & NA & NA & NA & NA \\
\hline III & $1856.939(0.000-2.6481 \mathrm{E} 61)$ & 0.912 & NA & NA & NA & NA \\
\hline IV & $3401.073(0.000-4.837 \mathrm{E}+61)$ & 0.905 & NA & NA & NA & NA \\
\hline Unknown & $1933.002(0.000-2.751 E+61)$ & 0.912 & NA & NA & NA & NA \\
\hline \multicolumn{7}{|l|}{ T staging } \\
\hline $\mathrm{T} 1$ & Reference & & Reference & NA & Reference & NA \\
\hline $\mathrm{T} 2$ & $0.759(0.355-1.621)$ & 0.477 & $0.794(0.335-1.882)$ & 0.600 & $0.872(0.365-2.082)$ & 0.757 \\
\hline $\mathrm{T} 3$ & $0.890(0.476-1.662)$ & 0.714 & $0.921(0.430-1.973)$ & 0.832 & $1.007(0.481-2.108)$ & 0.985 \\
\hline Unknown & $1.047(0.576-1.902)$ & 0.880 & $0.687(0.315-1.499)$ & 0.346 & $0.697(0.324-1.503)$ & 0.358 \\
\hline N staging & & & & & & \\
\hline N0 & Reference & & Reference & NA & Reference & NA \\
\hline N1 & $1.203(0.779-1.856)$ & 0.405 & $1.165(0.690-1.965)$ & 0.568 & $1.183(0.697-2.011)$ & 0.533 \\
\hline Unknown & $1.473(0.975-2.225)$ & 0.066 & $1.013(0.609-1.685)$ & 0.960 & $1.006(0.601-1.684)$ & 0.981 \\
\hline Histology & & & & & & \\
\hline Epithelial neoplasms & Reference & & Reference & & Reference & NA \\
\hline Gonadal neoplasms & $0.324(0.045-2.323)$ & 0.262 & $0.192(0.024-1.508)$ & 0.117 & $0.176(0.022-1.392)$ & 0.100 \\
\hline Others & $0.696(0.323-1.499)$ & 0.355 & $0.793(0.302-2.079)$ & 0.637 & $0.976(0.376-2.535)$ & 0.960 \\
\hline Unknown & $1.293(0.824-2.030)$ & 0.264 & $0.592(0.336-1.043)$ & 0.070 & $0.606(0.342-1.072)$ & 0.085 \\
\hline Treatment & & & & & & \\
\hline Others & Reference & & NA & NA & Reference & \\
\hline CSR & $0.209(0.112-0.390)$ & $<0.0001$ & NA & NA & $0.104(0.045-0.236)$ & $<0.0001$ \\
\hline RS & $0.555(0.136-2.275)$ & 0.414 & NA & NA & $0.350(0.069-1.783)$ & 0.206 \\
\hline SC & $0.224(0.081-0.620)$ & 0.004 & NA & NA & $0.061(0.018-0.212)$ & $<0.0001$ \\
\hline $\mathrm{RC}$ & $0.357(0.215-0.595)$ & $<0.0001$ & NA & NA & $0.231(0.124-0.429)$ & $<0.0001$ \\
\hline $\mathrm{R}$ & $0.863(0.443-1.683)$ & 0.666 & NA & NA & $0.710(0.307-1.641)$ & 0.423 \\
\hline $\mathrm{s}$ & $0.340(0.136-0.847)$ & 0.021 & NA & NA & $0.231(0.070-0.763)$ & 0.016 \\
\hline $\mathrm{C}$ & $0.502(0.285-0.884)$ & 0.017 & NA & NA & $0.310(0.158-0.608)$ & 0.001 \\
\hline Surgery & & & & & & \\
\hline $\mathrm{U} / \mathrm{BSO}$ & Reference & & Reference & & NA & NA \\
\hline Cytoreductive & $1.117(0.483-2.582)$ & 0.797 & $1.120(0.427-2.938)$ & 0.818 & NA & NA \\
\hline Others & $0.000(0.000-4.8509 \mathrm{E} 168)$ & 0.959 & $0.000(0.000-7.797 \mathrm{E}+183)$ & 0.961 & NA & NA \\
\hline No & $2.743(1.564-4.810)$ & $<0.0001$ & $3.141(1.433-6.886)$ & 0.004 & NA & NA \\
\hline Radiotherapy & & & & & & \\
\hline No & Reference & & Reference & & NA & NA \\
\hline Yes & $0.499(0.347-0.717)$ & $<0.0001$ & $0.817(0.462-1.442)$ & 0.485 & NA & NA \\
\hline Chemotherapy & & & & & & \\
\hline No & Reference & & Reference & & NA & NA \\
\hline Yes & $0.3720 .260-0.531()$ & $<0.0001$ & $0.298(0.180-0.495)$ & $<0.0001$ & NA & NA \\
\hline $\begin{array}{l}\text { Radiation Sequence } \\
\text { with surgery }\end{array}$ & & & & & & \\
\hline Others & Reference & & Reference & & NA & NA \\
\hline RBS & $0.365(0.090-1.481)$ & 0.158 & $1.283(0.247-6.660)$ & 0.767 & NA & NA \\
\hline RAS & NA & NA & NA & NA & NA & NA \\
\hline RBAS & $0.417(0.250-0.697)$ & 0.001 & $1.151(0.536-2.473)$ & 0.718 & NA & NA \\
\hline Brain Met & & & & & & \\
\hline
\end{tabular}




\begin{tabular}{|c|c|c|c|c|c|c|}
\hline \multirow[t]{2}{*}{ Variables } & \multicolumn{2}{|l|}{ Univariate } & \multicolumn{2}{|c|}{ Multivariate in model 1} & \multicolumn{2}{|c|}{ Multivariate in model 2} \\
\hline & HR (95\%CI) & P value & HR (95\%CI) & P value & HR $(95 \% \mathrm{CI})$ & P value \\
\hline No & Reference & & Reference & & Reference & \\
\hline Yes & $1.327(0.943-1.866)$ & 0.104 & $1.600(1.042-2.458)$ & 0.032 & $1.653(1.076-2.541)$ & 0.022 \\
\hline \multicolumn{7}{|l|}{ Bone Met } \\
\hline No & Reference & & Reference & & Reference & \\
\hline Yes & $1.186(0.780-1.805)$ & 0.425 & $0.951(0.554-1.630)$ & 0.854 & $1.040(0.596-1.814)$ & 0.891 \\
\hline Unknown & $1.877(0.999-3.528)$ & 0.050 & $0.977(0.307-3.112)$ & 0.969 & $0.974(0.297-3.193)$ & 0.965 \\
\hline \multicolumn{7}{|l|}{ Liver Met } \\
\hline No & Reference & & Reference & & Reference & \\
\hline Yes & $1.756(1.187-2.598)$ & 0.005 & $1.899(1.119-3.223)$ & 0.018 & $1.941(1.140-3.303)$ & 0.015 \\
\hline Unknown & $1.995(0.999-3.987)$ & 0.050 & $1.506(0.535-4.236)$ & 0.438 & $1.584(0.556-4.508)$ & 0.389 \\
\hline \multicolumn{7}{|l|}{ Lung Met } \\
\hline No & Reference & & Reference & & Reference & \\
\hline Yes & $1.834(1.274-2.641)$ & 0.001 & $1.374(0.826-2.284)$ & 0.221 & $1.305(0.785-2.169)$ & 0.305 \\
\hline Unknown & $1.949(0.934-4.068)$ & 0.075 & $1.500(0.455-4.941)$ & 0.505 & $1.436(0.438-4.712)$ & 0.550 \\
\hline \multicolumn{7}{|l|}{ CA125 } \\
\hline Normal & Reference & & Reference & NA & Reference & NA \\
\hline Elevated & $2.338(0.850-6.428)$ & 0.100 & $1.543(0.502-4.747)$ & 0.449 & $1.502(0.488-4.621)$ & 0.478 \\
\hline Unknown & $2.063(0.747-5.702)$ & 0.163 & $1.398(0.457-4.275)$ & 1.398 & $1.387(0.452-4.259)$ & 0.567 \\
\hline \multicolumn{7}{|c|}{ Insurance situation } \\
\hline No & Reference & & Reference & & Reference & \\
\hline Yes & $0.491(0.274-0.878)$ & 0.017 & $0.701(0.323-1.522)$ & 0.369 & $0.704(0.324-1.531)$ & 0.376 \\
\hline Unknown & $0.644(0.210-1.980)$ & 0.443 & $0.897(0.234-3.446)$ & 0.875 & $0.938(0.244-3.607)$ & 0.926 \\
\hline \multicolumn{7}{|l|}{ Residence type } \\
\hline Rural & Reference & & Reference & NA & Reference & NA \\
\hline Urban & $0.599(0.177-2.023)$ & 0.409 & $0.956(0.202-4.525)$ & 0.955 & $0.892(0.182-4.373)$ & 0.888 \\
\hline Metropolitan & $0.428(0.135-1.359)$ & 0.150 & $0.587(0.129-2.677)$ & 0.491 & $0.535(0.112-2.547)$ & 0.432 \\
\hline Unknown & NA & NA & NA & NA & NA & NA \\
\hline
\end{tabular}

\section{Discussion}

This study described the incidence and survival of the ovarian carcinoma patients with brain metastases at intial diagnosis based on the SEER database. The present study was the first time investigating both predictors and prognostic factors of de novo OCBM. Noteworthily, this population-based study also firstly employed the PSM analysis method to evaluate the role of brain metastases in ovarian carcinoma. BM was considered to be a rare and late event in ovarian carcinoma (3), it usually followed with high mortality, poor prognosis, causing colossal health burden and expensive medical costs (17). Although several systematic reviews and articles had been published upon the topic of ovarian cancer brain metastases $(1,3,14,18)$, sufficient evidence had not been found to provide clear guidelines on proper treatment, let alone the de novo brain metastases of ovarian carcinoma. In this circumstance, early detection and comprehensive treatment were of great significance, for it may alter the natural progression and improve overall survival. Thus, it is necessary to study OCBM patients in a large-scale cohort.

We found that $0.30 \%$ of patients with brain metastases at initial diagnosis of ovary cancer, and $12.02 \%$ was with metastatic disease at diagnosis, which were similar to previous study(19), and a little lower than some researches $(6,7,10,17,20-22)$. This may be because they counted not only patients with brain metastases at the time of diagnosis of ovarian cancer, but also patients with subsequent brain metastases. We identified predictors of OCBM using multivariate logistic regression. This study found that the unspecified histology, absence of surgery, no chemotherapy, and more extracranial metastasis sites increased the risk of developing OCBM in the entire cohort, which was similar to previous studies $(8,14$, 18, 20, 23-25). However, Cohen et al. (10) held the opposite view that there was no association between the $\mathrm{BM}$ and the presence of other extracranial metastases.

Moreover, we also found that patients with bilateral laterality were more likely to be OCBM, which had never been reported before as we knew. Intriguingly, radiotherapy was found to be a higher risk of metastatic diseases to any site, including brain metastases, which might due to the fact that OCBM patients are usually in advanced disease when receiving radiation therapy as palliative treatment. However, only surgery and chemotherapy treatment had a lower risk to be OCBM, and our study did not show that positive or negative of CA125 elevation associated with OCBM, which were consistent to the research published before(18, 26), however, was different with Divine et al. (25). These researches $(1,8$, 27 ) showed that elder age, residence type and ethnicity were the risk factors for brain metastases, but in our study, these variables only achieved significance on the univariate logistic analysis.

This study indicated that bilateral laterality, unspecified histology, absence of surgery or chemotherapy, more extracranial metastasis might suffer a higher likelihood to be brain metastases 
among OC patients. Thus, these high-risk people might require a further examination at the initial diagnosis. Besides, patients without insurance should be encouraged to get screening regularly.

Furthermore, we found that divorced marriage, absence of surgery, absence of chemotherapy, and no insurance harmed overall survival among OCBM upon multivariate logistic regression analysis. And histology, radiotherapy and insurance status were not associated with prognosis which were similar to the previous study (28-30). We supposed that divorced patients went through more sufferings during the failed marriage, getting less treatment due to lacking their spouses' support, leading to poor survival. However, the unmarried status like single and widowed did not achieve statistical significance in overall survival in this study, which differed from the previous research. The extracranial metastases sites which showed decreased survivals had been reported $(10,15,31)$, while in our study, extracranial metastases showed poorer survival among the entire cohort, but not in the OCBM subset. However, the reason remains unknown which needs further exploration.

To obtain satisfactory cytoreductive surgery and sensitive chemotherapy are the common treatment strategy for ovarian cancer $(32,33)$, which also applies to OCBM patients in this study. Surgery, radiation, and chemotherapy are the major treatment methods to prolong the patients' survival. Mostly, surgery supplemented with radiation therapy is a common choice. In our study, chemotherapy was the most common treatment in 89 patients $(46.1 \%)$, which is slightly different from the previous research, for these had radiotherapy as the mostly used treatment (24). However, it is still controversial on the effect of chemotherapy for many chemotherapeutic drugs that cannot permeate the blood-brain barrier (BBB), which drastically limits the effectiveness. And some concerns that the use of systemic chemotherapy may corrupt the BBB. Thus, radiotherapy remains top choice for OC treatment, except for the brain metastases, which in turn helps to explain the former difference. There are two types of radiotherapy, which are commonly used, including stereotactic radiosurgery (SRS) and whole-brain radiotherapy (WBRT). Some authors $(24,34)$ reported that WBRT could cause physical and cognitive decline. A systemic retrospective study (35) confirmed that SRS had a better effect on the treatment of OCBM, with more adequately measured lesions, less-lethal of healthy cells as well as less adverse responses, compared to the WBRT. Besides, recent studies have shown that systemic chemotherapy for brain metastases showed favorable responses and prolonging their survival $(6,17,18)$.
In model 1, the median survival time of OCBM patients decreased 2.5 months from chemotherapy to no chemotherapy $(\mathrm{P}<0.001)$. Moreover, median survival time decreased 10 months from U/BSO surgery to no surgery $(\mathrm{P}<0.001)$ and the prognosis for U/BSO surgery looks similar to the cytoreductive surgery $(\mathrm{P}>0.05)$. The median survival time from no radiotherapy to radiotherapy increased by 2.5 months $(\mathrm{P}<0.001)$. Besides, compared with surgery only $(4.5$ mo) $(\mathrm{P}>0.05)$ or chemotherapy only $(3.0 \mathrm{mo})$ $(\mathrm{P}=0.031)$, patients who acquired surgery plus chemotherapy seemed to have longer median survival (20 mo). In model 2, median survival time of OCBM patients increased 12 months from others to RSC, 2 months from others to RS, decreased 20 months from SC to others, 3 months from $C$ to others $(P>0.05), 4.5$ months from $S$ to others $(P>0.05)$, and there was little difference from others to RS or R. This result indicated that patients receiving multimodal therapy had significant benefits on survival. According to the results above, OCBM patients benefit more from chemotherapy and surgery, especially a combination of two, but radiotherapy achieved no statistical significance in overall survival analysis. The median survival of SC was the best, while patients with "others" treatment had the worst prognosis. The present study showed SC had the best prognosis, followed by CSR and RC among the comprehensive treatments for OCBM, but RS showed no statistical difference for overall survival, which might be meaningful for clinical practice. What's more, we found similar results in our PSM-matched analysis: in model 1, divorced marital status, absence of treatment including surgery and chemotherapy, liver met, and lung met were associated with more reduced overall survival among OCBM patients. Likewise, in model 2, single, divorced marital status showed poor prognosis, and combined treatment including CSR, SC, and RT was significantly associated with improved OS. Surgery or chemotherapy could also considerably reduce the risk of OCBM death. However, radiotherapy did not show statistical significance in improving the overall survival among OCBM patients based on the PSM-matched analysis.

\section{Limitations}

Although this study is the large-scale multi-center study, but it has some limitations. Firstly, we only know the information of four metastatic sites, including the liver, bone, lung, and brain. This database has not included information on other metastatic sites, such as peritoneal metastases. Besides, we only knew the information of synchronous metastasis to the brain, consisting of a 
small part regarding those who might develop metachronous metastasis afterward. Secondly, some well-established covariates of survival such as comorbidities or performance status or even some tumor-related data such as stage, the types and numbers of brain metastases, the size of tumor, mammography screening, and treatment for comorbidities are not available in the database. Thirdly, the SEER database has not recorded the morbidity and mortality after treatment. Lastly, residence type was labeled at a county level, rather than a patient level, which may affect the results of the analysis.

This study firstly conducted a population-based analysis of OCBM patients as well as PSM-based analysis, to our knowledge. It provided valuable advice for patients with higher risk of OCBM to consider using MRI assessment. Besides, we also analyzed the prognostic factors of OCBM in the research. Additionally, the study compared the significance of different therapies for OCBM patients and provided recommendations for their clinical treatment. Moreover, this large-scale study used an efficient statistical method to emphasize the reliability of the impact of de novo brain metastases on survival in patients with OC.

\section{Supplementary Material}

Supplementary figures and tables.

http://www.jcancer.org/v11p4625s1.pdf

\section{Acknowledgments}

We thanked that all staff of the National Cancer Institute of the United States and at Information management Services, Inc., who have been contributing to the Surveillance, Epidemiology, and End Results (SEER) Program. Also we would like to thank Mr. Xu for the statistical consultation.

\section{Funding}

This work was supported by the Science and Technology Planning Projects of Guangzhou City (2016201604030009, 201707010005), Guangdong Natural Science Foundation (2016A030313250, 2017A030313711).

\section{Author Contributions}

XS and ML designed the study. XS, LZ and GQ acquired the data. XS, LX, and LZ analyzed the data. XS, GQ and LZ organized the manuscript. GQ, LZ and ML reviewed the papers and revised the manuscript. All the authors (XS, LZ, GQ, LW, LX, and ML) have read and approved the final manuscript. All the authors agreed to be responsible for all aspects of the work.

\section{Data Availability Statement}

All datasets analyzed for this study are available in the SEER database, https:// seer.cancer.gov/data/.

\section{Ethics Statement}

The SEER was a public-use dataset, of which the informed consent was waived. Moreover, this study was considered exempt from review by the Ethics Board of The Seventh Affiliated Hospital, Sun Yat-sen University.

\section{Competing Interests}

The authors have declared that no competing interest exists.

\section{References}

1. Torre LA, Trabert B, Desantis CE, et al. Ovarian Cancer Statistics, 2018. CA-Cancer J Clin. 2018; 68(4): 284-96.

2. Siegel RL, Miller KD, Jemal A. Cancer Statistics, 2018. CA-Cancer J Clin. 2018; 68(1): 7-30.

3. Achrol AS, Rennert RC, Anders C, et al. Brain metastases. Nat Rev Dis Primers. 2019; $5: 26$.

4. Weidle UH, Birzele F, Kollmorgen G, et al. Mechanisms and Targets Involved in Dissemination of Ovarian Cancer. Cancer Genomics Proteomics. 2016; 13(6): 407-23.

5. Jelovac D, Armstrong DK. Recent Progress in the Diagnosis and Treatment of Ovarian Cancer. CA-Cancer J Clin. 2011; 61(3): 183-203.

6. Deng K, Yang CY, Tan QL, et al. Sites of distant metastases and overall survival in ovarian cancer: A study of 1481 patients. Gynecologic Oncology. 2018; 150(3): 460-65

7. Stasenko M, Cybulska P, Feit N, et al. Brain metastasis in epithelial ovarian cancer by BRCA1/2 mutation status. Gynecologic Oncology. 2019; 154(1): 144-49.

8. Ratner E, Bala M, Louie-Gao M, et al. Increased risk of brain metastases in ovarian cancer patients with BRCA mutations. Gynecologic Oncology. 2019; 153(3): 568-73

9. Cormio G, Loizzi V, Falagario M, et al. Central Nervous System Metastases From Epithelial Ovarian Cancer Prognostic Factors and Outcomes. Int J Gynecol Cancer. 2011; 21(5): 816-21.

10. Cohen ZR, Suki D, Weinberg JS, et al. Brain metastases in patients with ovarian carcinoma: prognostic factors and outcome. J Neuro-Oncol. 2004; 66(3): 313-25.

11. D'andrea G, Roperto R, Dinia L, et al. Solitary cerebral metastases from ovarian epithelial carcinoma: 11 cases. Neurosurg Rev. 2005; 28(2): 120-23.

12. Cervoni L, Gagliardi FM. Cerebral metastasis from ovarian carcinoma. Some observations about treatment. Minerva ginecologica. 1999. 51(1-2): 35-7.

13. Pectasides D, Aravantinos G, Fountzilas G, et al. Brain Metastases from epithelial ovarian cancer. The Hellenic cooperative oncology group (HeCOG) experience and review of the literature. Anticancer Res. 2005; 25(5): 3553-58.

14. Piura E, Piura B. Brain metastases from ovarian carcinoma. ISRN oncology. 2011; 2011: 527453

15. Zhang C, Guo X, Peltzer K, et al. The prevalence, associated factors for bone metastases development and prognosis in newly diagnosed ovarian cancer: a large population based real-world study. J Cancer. 2019; 10(14): 3133-39.

16. Sun ZP, Zheng H, Yu J, et al. Liver Metastases in Newly Diagnosed Gastric Cancer: A Population-Based Study from SEER. J Cancer. 2019; 10(13): 2991-3005

17. Liu $\mathrm{P}$, Liu W, Feng $\mathrm{YH}$, et al. Brain metastasis from ovarian clear cell carcinoma A case report. Medicine. 2019; 98(3): 6

18. Pakneshan S, Safarpour D, Tavassoli F, et al. Brain metastasis from ovarian cancer: a systematic review. J Neuro-Oncol. 2014; 119(1): 1-6.

19. Kolomainen DF, Larkin JMG, Badran M, et al. Epithelial ovarian cancer metastasizing to the brain: A late manifestation of the disease with an increasing incidence. J Clin Oncol. 2002; 20(4): 982-86.

20. Marchetti C, Ferrandina G, Cormio G, et al. Brain metastases in patients with EOC: Clinico-pathological and prognostic factors. A multicentric retrospective analysis from the MITO group (MITO 19). Gynecologic Oncology. 2016; 143(3): 532-38.

21. Gien LT, Kwon JS, D'souza DP, et al. Brain metastases from endometrial carcinoma: a retrospective study. Gynecologic Oncology. 2004; 93(2): 524-28.

22. Thomakos N, Diakosavvas M, Machairiotis N, et al. Rare Distant Metastatic Disease of Ovarian and Peritoneal Carcinomatosis: A Review of the Literature. Cancers. 2019; 11(8): 17.

23. Pectasides D, Pectasides $M$, Economopoulos T. Brain metastases from epithelial ovarian cancer: A review of the literature. Oncologist. 2006; 11(3): $252-60$ 
24. Lukas RV, Gabikian P, Garza M, et al. Treatment of Brain Metastases. Oncology. 2014; 87(6): 321-29.

25. Divine LM, Kizer NT, Hagemann AR, et al. Clinicopathologic characteristics and survival of patients with gynecologic malignancies metastatic to the brain. Gynecologic Oncology. 2016; 142(1): 76-82.

26. Nieder C, Spanne O, Mehta MP, et al. Presentation, Patterns of Care, and Survival in Patients With Brain Metastases What Has Changed in the Last 20 Years? Cancer. 2011; 117(11): 2505-12.

27. Barnholtz-Sloan JS, Sloan AE, Davis FG, et al. Incidence proportions of brain metastases in patients diagnosed (1973 to 2001) in the metropolitan Detroit cancer surveillance system. J Clin Oncol. 2004; 22(14): 2865-72.

28. Mahdi H, Kumar S, Munkarah AR, et al. Prognostic impact of marital status on survival of women with epithelial ovarian cancer. Psycho-Oncol. 2013; 22(1): 83-88.

29. Ibfelt EH, Dalton SO, Hogdall C, et al. Do stage of disease, comorbidity or access to treatment explain socioeconomic differences in survival after ovarian cancer? - A cohort study among Danish women diagnosed 2005-2010. Cancer Epidemiol. 2015; 39(3): 353-59.

30. Aizer AA, Chen MH, Mccarthy EP, et al. Marital Status and Survival in Patients With Cancer. J Clin Oncol. 2013; 31(31): 3869-76.

31. Mahmoud-Ahmed AS, Kupelian PA, Reddy CA, et al. Brain Metastases from Gynecological Cancers: Factors that Affect Overall Survival. Technol Cancer Res Treat. 2002; 1(4): 305-10.

32. Langyel E. Ovarian Cancer Development and Metastasis. American Journal of Pathology. 2010; 177(3): 1053-64.

33. Aletti GD, Gallenberg MM, Cliby WA, et al. Current management strategies for ovarian cancer. Mayo Clin Proc. 2007; 82(6): 751-70.

34. Aoyama $\mathrm{H}$, Tago $\mathrm{M}$, Kato $\mathrm{N}$, et al. Neurocognitive function of patients with brain metastasis who received either whole brain radiotherapy plus stereotactic radiosurgery or radiosurgery alone. Int J Radiat Oncol Biol Phys. 2007; 68(5): 1388-95.

35. Choo BA, Walji N, Spooner D, et al. Prolonged Relapse-Free Survival in Two Patients With an Isolated Brain Metastasis From Epithelial Ovarian Carcinoma. J Clin Oncol. 2010; 28(17): E271-E72. 